\title{
Pair Density Waves in coupled doped two-leg Ladders
}

\author{
Javier Almeida, ${ }^{1}$ Guillaume Roux, ${ }^{2}$ and Didier Poilblanc ${ }^{1}$ \\ ${ }^{1}$ Laboratoire de Physique Théorique, CNRS and Université de Toulouse, UPS, (IRSAMC), F-31062 Toulouse, France \\ ${ }^{2}$ Univ Paris-Sud, Laboratoire de Physique Théorique et Modèles Statistiques, \\ UMR8626, Orsay F-91405, France; CNRS, Orsay, F-91405, France.
}

(Dated: June 15, 2018)

\begin{abstract}
Motivated by Resonant X-ray scattering experiments in cuprate ladder materials showing charge order modulation of period $\lambda=3$ and 5 at specific hole densities, we investigate models involving the electronic t-J ladders and bosonic chains coupled via screened Coulomb repulsion. Extensive density matrix renormalization group calculations applied to the ladders/chains supplemented by a self-consistent mean-field treatment of the inter-ladder/chain coupling provide quantitative estimates of the charge order for $\lambda=3,4$ and 5. As previously proposed, such patterns correspond to the emergence of pair density waves which stem from the strong electronic correlations. We comment on the existence of a $\lambda=4$ modulation not seen so far in experiment.
\end{abstract}

PACS numbers: 71.45.Lr, 75.40.Mg, 74.72.Gh

It is fascinating that electron correlations alone could lead to unconventional pairing, eventually leading to superconducting or other exotic phases such as pair crystals, e.g. in the two-dimensional (2D) high critical-temperature cuprate superconductors. The quasi one-dimensional (1D) two-leg ladder material $\mathrm{Sr}_{14-x} \mathrm{Ca}_{x} \mathrm{Cu}_{24} \mathrm{O}_{41}$ (SCCO) is another remarkable example 1 of strongly correlated material displaying both unconventional superconducting and charge-density wave (CDW) phases. It is a layered compound with intercalated chain and two-leg ladder subunits. Under doping and high hydrostatic pressure, the ladder layers exhibit superconductivity $\stackrel{2}{=}$ For $x=0$, the SCCO is intrinsically hole-doped and showed a charge modulation within the chain layers, $\underline{3}$ which later was attributed to a buckling of the chains. ${ }^{4}$ On the other hand, another set of experiments revealed evidence of commensurate charge modulations, with periods $\lambda$ of 3 - and 5-lattice spacings along the ladders, which bear their origin in the interactions between electrons ${ }^{5-7}$ Surprisingly, these experiments did not find modulations with period $\lambda=4$ in contrast to theoretical results on isolated ladders, ${ }^{8,9}$ hence suggesting that the frustrated nature of the zig-zag inter-ladder coupling, forming a trellis lattice (see Fig. 11), has a crucial role.

In this Rapid Communication, we carry out a systematic investigation of the $2 \mathrm{D}$ charge ordering in models of coupled chains of hard-core bosons (HCB) and electronic ladders. We use a combination of the density matrix renormalization group $^{10}$ (DMRG) technique to solve the quasi-1D subsystems and a mean-field (MF) treatment of the screened Coulomb repulsion between them. Even though our main focus is the physics of doped spin ladders, the simpler hard-core boson model is also of great interest since (i) it displays similar density fluctuations and (ii) it is closely related to the ladder model in the limit of very large exchange rung coupling. 11 Results from these general models are lastly compared with other possible interpretations of the experiments.

Models and their 1D charge fluctuations - We start by recalling the behavior of charge fluctuations in an isolated doped

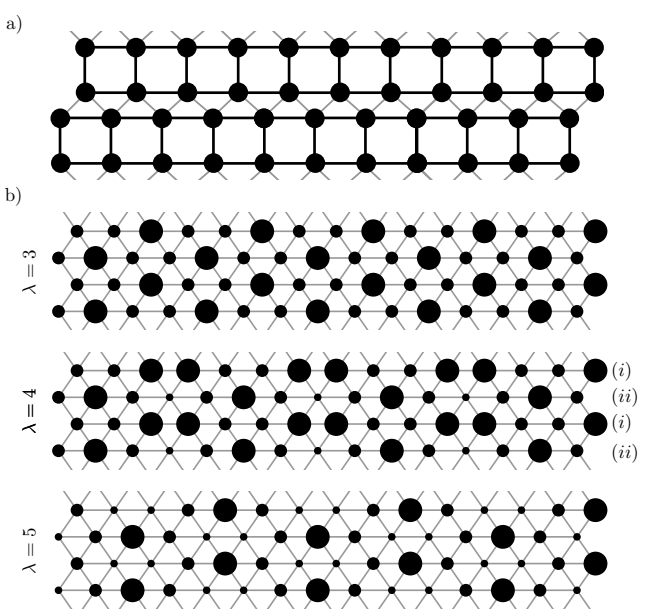

FIG. 1: (a) Sketch of coupled ladders in a layer of the SCCO compound. (b) Proposed CDW modulations for $\lambda=3,4,5$ (as the electronic density is identical on both leg, only one is represented).

two-leg ladder that we describe by the isotropic t-J model:

$$
\mathcal{H}=-t \sum_{\langle i, j\rangle, \sigma} \mathcal{P}\left[c_{i, \sigma}^{\dagger} c_{j, \sigma}+\text { h.c. }\right] \mathcal{P}+J \sum_{\langle i, j\rangle}\left[\mathbf{S}_{i} \cdot \mathbf{S}_{j}-\frac{1}{4} n_{i} n_{j}\right]
$$

where $\mathcal{P}$ are Gutzwiller projectors, $c_{i, \sigma}$ the electron annihilation operators with spin $\sigma$ at site $i, n_{i}$ the density operator and $\mathbf{S}_{i}$ the spin operator. The antiferromagnetic spin exchange $J$ is set to $0.35 t$, a typical value for cuprates. The model has a spin gap that survives to finite doping $\delta, 12$ beyond $\delta=1 / 3$. Its low-energy physics is described by a single charge mode $\phi(x)$ governed by a Luttinger Liquid (LL) effective Hamiltonian ${ }^{13}$ :

$$
\mathcal{H}=\int \frac{d x}{2 \pi}\left[u K(\pi \Pi(x))^{2}+\frac{u}{K}(\nabla \phi(x))^{2}\right],
$$

with $\Pi(x)$ the canonically conjugated field. Due to the presence of a spin gap, the leading charge fluctuations are not the usual $2 k_{F}$ ones but the $4 k_{F}$ ones. In the following, we will denote this wave-vector $q=2 \pi n \equiv 4 k_{F}$, with $n$ the electronic density $(\delta=1-n=1 / \lambda)$. The density fluctuations 
have a power-law decay $\langle n(x) n(0)\rangle_{c} \sim \cos (q x) x^{-2 K}$ governed by the Luttinger parameter $K$. Depending on $K$, there is a competition between a $4 k_{F}$-CDW $(K<1 / 2)$ and a $d$ wave superconducting $(K>1 / 2)$ phase: the phase diagram has been obtained numerically ${ }^{8,14}$ For the commensurate fillings $n=3 / 4$ and $n=1 / 2$, two different kinds of ordered phases appear ${ }^{8.9}$ In particular, strong interactions yield a commensurate CDW for $\lambda=4$ but no signatures of translational symmetry breaking was observed ${ }^{9}$ for $\lambda=3$ or 5 .

Furthermore, some insight into the 2D CDW locking will be obtained using the 1D following HCB t-V model:

$$
\mathcal{H}=-t \sum_{i}\left[b_{i}^{\dagger} b_{i+1}+\text { h.c. }\right]+V \sum_{i} n_{i} n_{i+1}
$$

with $b_{i}$ the hard-core boson annihilation operator and $V$ the nearest-neighbor repulsion. The choice is motivated by the analogy between hole pairs and bosons .11 For isotropic ladder, a more rigorous mapping would also involve additional bosons for triplets. 15 However, our bosonic model has the same effective Hamiltonian (11) as the t-J ladder and its leading density fluctuations are $2 k_{F}$ ones with exponent $2 K$. In terms of the boson density $n=1 / \lambda$, we have exactly the same wave-vector $q=2 \pi n \equiv 2 k_{F}$ as for the ladders. In spite of this direct analogy, the behavior of $K$ is different in this model ${ }^{13}$

Mean-field treatment and CDW patterns - We now turn to the effect of coupling these quasi-1D charge fluctuations via screened Coulomb repulsion (we neglect other couplings, such as particle or pair hoppings). According to the lattice structure of SCCO and recent ab-initio calculations, $\frac{16}{-}$ we consider that the main interactions between adjacent ladders are along the grey bonds of Fig. 1a, the magnitude of which is denoted by $V_{\perp}$. Using a MF approximation, these couplings boil down to a chemical potential term $-\mu_{i} n_{i}$ with

$$
\mu_{i}=-V_{\perp} \sum_{j=\text { neighbor }(i)}\left\langle n_{j}\right\rangle .
$$

The local density $\left\langle n_{j}\right\rangle$ is then determined self-consistently. For $\lambda=3$ and 5 , the patterns which minimize the energy are naturally site-centered CDW shifted by $\pi$ (see Fig. 1b) corresponding to

$$
\left\langle n_{j}\right\rangle=n\left[1+\rho_{q} \cos (q(j-\varphi))+\rho_{2 q} \cos (2 q j)\right]
$$

with $\varphi=0$. Notice that there actually is only one harmonic for $\lambda=3$ and two for $\lambda=5$ with the particularity that $2 q \equiv q / 2$ (hence $2 q=8 k_{F} \equiv 2 k_{F}$ in ladders). In the limit of small $V_{\perp}$, the MF scheme is actually equivalent to static RPA as the condition for the order to develop is $1=z V_{\perp} \gamma(q) \chi_{1 D}(q)$, with $\gamma(q)$ a geometrical prefactor, $z$ the coordination number and $\chi_{1 D}(q)$ the static charge susceptibility of the isolated quasi-1D system. Classical configurations with one particle each $\lambda$ site, expected in the large $V_{\perp}$ limit, are also described by Eq. (3) so that the MF scheme interpolates between the perturbative (RPA) regime to the nonperturbative (classical) one. In both repulsive models under study, for which $K<1, \chi_{1 D}(q)$ diverges $^{13}$ and the order builds up as soon as $V_{\perp}$ is branched. Focusing now on the

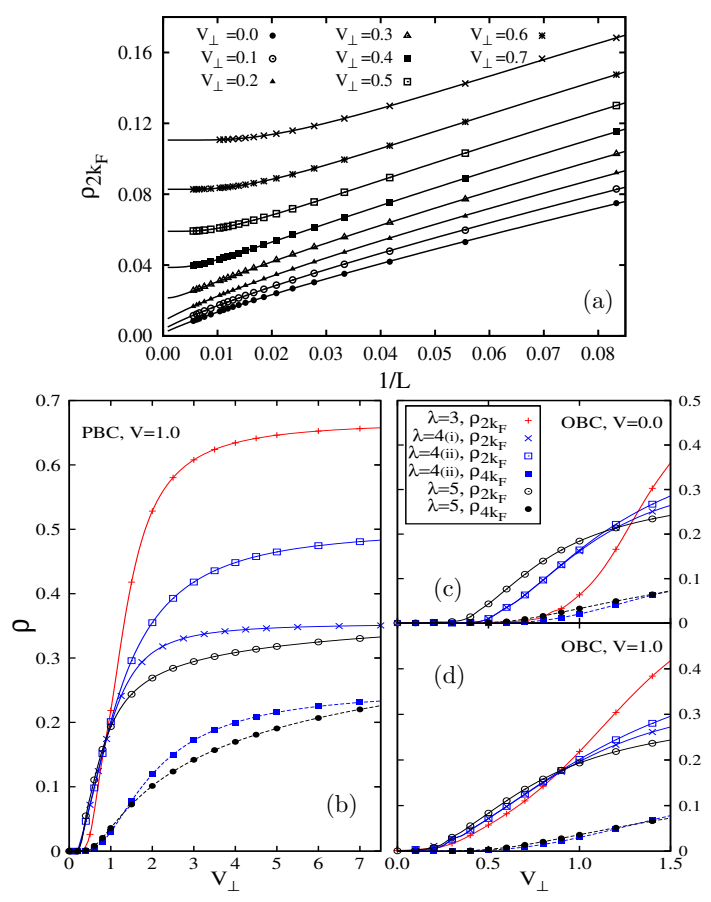

FIG. 2: (Color online) HCB model: (a) Finite size scaling of $\rho_{q}$ for $\lambda=3$ and $V=1$. (b) results with PBC showing the large $V_{\perp}$ classical limit. (c-d) Extrapolated results with OBC showing the opening of the order for $\lambda=3,4,5$ when $V=0$ and $V=1$.

classical limit when $\lambda=4$, the frustration of the zig-zag couplings makes it impossible to accommodate two chains with one particle every four sites, as it happens for the square lattice. Striped configurations could be realized but we actually found that the most stable pattern is again two shifted $\mathrm{CDW}^{21}$ of the type (3) (see Fig. 1 1 ) which can yet have different amplitudes, contrary to the $\lambda=3,5$ cases. The CDW (i) is bondcentered $(\varphi=1 / 2)$ while the other (ii) is site-centered. Here again, the RPA argument gives that the order develops at any finite $V_{\perp}$. Numerical calculations are performed taking one finite quasi-1D system with open boundary conditions and solved by DMRG, keeping 800 states $^{22}$, embedded between two infinite ones displaying the pattern (3). When $\lambda=4$, the two patterns (i) and (ii) are solved simultaneously. In the selfconsistent procedure, the $\rho_{q}$ 's are sampled in the bulk and the convergence criteria is to have a relative energy error smaller than $10^{-5}$, for the which the density patterns are converged.

HCB model - We start the discussion with the HCB model. As it can be solved efficiently, we use both periodic (PBC) and open boundary conditions (OBC) to check the finite size effects. For PBC, a finite-size chain does not display an order below a certain value $V_{\perp}^{c}(L)$. This can be understood in terms of a correlation length $\xi$ larger than $L$, making the scaling of the order parameter with $L$ difficult with PBC. With OBC, there always is some charge fluctuations at the edges which decay toward the bulk value, the so-called Friedel oscillations. If the field $\phi$ is not pined, these oscillations are well fitted by 


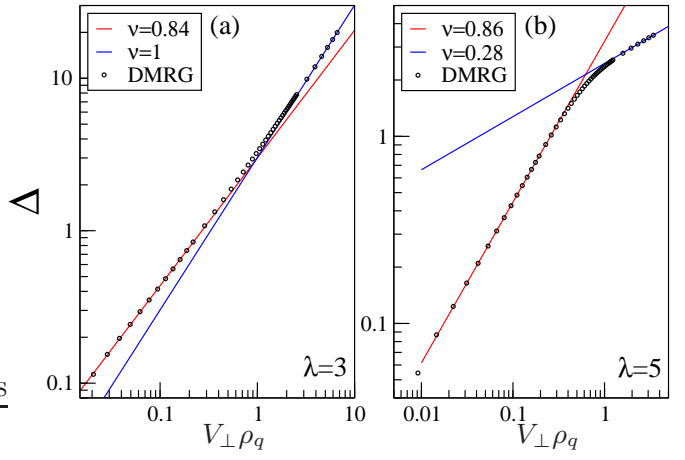

FIG. 3: (Color online) Scaling of the opening of the gap for $\lambda=3$ (a) and $\lambda=5$ (b) in the HCB model with $V=1$ (extrapolated data).

the following ansatz based on LL theory and symmetries:

$$
n(x)=n+A \frac{\cos q(x-(L+1) / 2)}{[\sin (\pi x /(L+1))]^{K}}
$$

which gives an access to the Luttinger parameter $K$ (the decay exponent of the $2 q$ harmonic is $4 K$ so that its contribution is negligible). When $\phi$ gets pined, the decay of the oscillations are exponentially suppressed, as $e^{-x / \xi}$. Using OBC, we start the MF iterations from the $V_{\perp}=0$ density profile that displays Friedel oscillations and, once convergence is reached, we scale the bulk value of $\rho_{q}$ using the following ansatz which interpolates between the two regimes:

$$
\rho_{q}(L)=\rho_{q}^{\infty}+B e^{-L / \xi} / L^{\alpha} .
$$

According to the results gathered in Fig. 2a, such an ansatz looks reasonable. In Fig. 2 (b-d), one finds the opening of the order through the behavior of its Fourier components $\rho_{q}$ for $\lambda=3,4,5$ both in the non-interacting $(V=0)$ and interacting $(V=1)$ regimes. As predicted by RPA, the $q$-component opens first while the harmonic at $2 q$ develops above $V_{\perp} \gtrsim 0.5$. Remarkably, $\rho_{2 q}$ hardly has finite-size effects. Notice that, for $\lambda=4$, the main Fourier components of the two inequivalent chains (i) and (ii) have the same magnitude as one may infer in the perturbative regime of the continuum limit. In the large $V_{\perp}$ limit, the Fourier components saturate close to their classical expectations for $\lambda=3$ and 4(i). For $\lambda=5$, the nearestneighbor repulsion is not sufficient to pin the bosons every five sites. We found that such a classical pattern can be realized by taking into account longer ranged interactions within the MF approximation (data not shown). In all cases, we conclude that the patterns of Fig. $1 \mathrm{~b}$, similar to a Wigner crystal of bosons, can be realized with a sizable order for sufficiently large yet short range interactions.

Although LL theory cannot give a quantitative prediction for the magnitude of $\rho_{q}$, it provides the scaling of the gap associated with the ordering. The one boson gap is defined as

$$
\Delta(L)=E_{0}(N+1)+E_{0}(N-1)-2 E_{0}(N)
$$

with $E_{0}(N)$ the ground-state energy with $N$ bosons. All energies are computed with the same external $\mu_{i}$ obtained from the
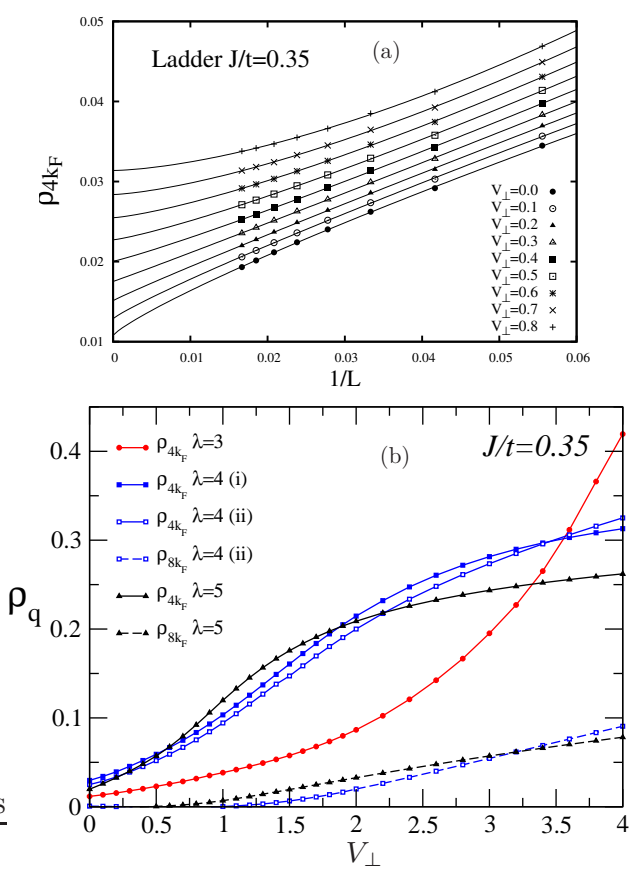

FIG. 4: (Color online) $t J$ model: (a) finite-size extrapolations of $\rho_{q}$ for $\lambda=3$. (b) Opening of the Fourier component with the interladder coupling $V_{\perp}$ (extrapolated data for $\lambda=3$ and 5). The $\lambda=4$ data are shown for $L=60$ and cannot be extrapolated (see text).

converged density pattern. In other words, we assume that the surrounding charge distribution of the neighboring chains is not perturbed by removing/adding one particle. Gaps are then extrapolated using $\Delta(L)=\Delta^{\infty}+B e^{-L / \xi} / L$. At the level of LL theory, the MF coupling yields a sine-Gordon term ${ }^{17}$ which generically reads

$$
g \int d x \cos \beta \phi(x) .
$$

Such a perturbation is relevant when $K<K_{c}=8 / \beta^{2}$. For the main harmonic $q$, one has $g \propto V_{\perp} \rho_{q}$ and $\beta=2$. Thus, we have $K_{c}=2$ so that when $\rho_{q} \neq 0$, one is deep into the massive phase of (6). In this regime, the gap scales ${ }^{23}$ as $\Delta \propto$ $g^{\nu}$ with $\nu=K_{c} / 2\left(K_{c}-K\right)=1 /(2-K)$. In Fig. 3a, we report the scaling of the gap for $V=1$ and $\lambda=3$ (for which there is no extra harmonic), suggesting an exponent $\nu \simeq 0.84$. A perturbative estimate of $K$ is $[1+V /(\pi t \sin (\pi n))]^{-1 / 2} \simeq$ 0.855 which gives $\nu=0.87$. A non-perturbative estimate of $K$ is deduced from fitting the density pattern using Eq. (4): we find $K \simeq 0.78$ and $\nu \simeq 0.82$. The agreement with the sineGordon predictions is consequently very good up to relatively large $V_{\perp}$ (for these particular set of parameters). Beyond this regime, one recovers $\nu=1$ which is expected when the $\lambda=3$ pattern saturates: DMRG results nicely interpolate between the two regimes. In Fig. 3 , similar results are found for $\lambda=$ 5 , excepting that the large $V_{\perp}$ exponent is much smaller $(\nu \simeq$ 0.28 ) because there is more room in the MF potential for the extra particle. The large $V_{\perp}$ is thus strongly dependent on the density and the range of the interactions. 
$t$ - $J$ ladders - We now turn to the more realistic case of doped ladders. First, we recall that, in an isolated ladder, Umklapp processes can bring a sine-Gordon term (6) with $\beta=8$ when $\lambda=4$. Even though the corresponding critical value $K_{c}=1 / 8$ is very small, signatures 8.9 of this $1 \mathrm{D}$ crystal has been found for small enough $J / t$. From Eq. (4), we get the following values of $K_{\lambda}$ for $J / t=0.35$ on a system with $L=120: K_{3} \simeq 0.55, K_{4} \simeq 0.42$ and $K_{5} \simeq 0.58$. We notice a few qualitative differences with the HCB model. Firstly, $K$ is smaller for $\lambda=4$ suggesting that this commensurability will lead to the larger order in the continuum limit. Secondly, the coordination number is now $z=2$ instead of $z=4$ for HCB. This roughly induces a factor two in the $V_{\perp}$ required to stabilize a sizable order. In Fig. $4 \mathrm{a}$, we give the finite size scaling of the Fourier amplitudes in the $\lambda=3$ case using (5). As $K \sim 0.5$, finite-size extrapolations are much harder. For $\lambda=3$ and 5, the fits give $\rho_{q}^{\infty} \simeq 0.01,0.02$ which is within numerical accuracy. For $\lambda=4$, there is a finite size effect which makes the extrapolation impossible: for the (ii) chain, which pattern is ABCBABC..., we must take a ladder with one more rung to preserve the reflection symmetry about the center. Contrary to the HCB model (for which there is no issue with the finite size scaling), the minima of the density are gradually shifted by one site between the edges and the middle. The consequence is that the pattern (3) becomes frustrating at the edges, lowering the overall order on small systems. We observe in Fig. 4b that the order increases slower for $\lambda=3$ than for $\lambda=4,5$. This is qualitatively different from the HCB model with $V=1$. We may attribute this difference to the effective behavior of pairs of holes in the ladder. In fact, hole pairs in isotropic doped ladders can be viewed as a hole pair resonating with a singlet on a plaquette. The center of mass of the pair can live on a rung or at the center of the plaquette so that their effective hard-core bosonic model has twice the number of sites. 11 We suggest that the slower opening of the $\lambda=3$ order is related to the extension of the hole pairs which favors overlapping at large doping. This (rel- ative) reduction of the density fluctuations with doping even leads to the absence ${ }^{9}$ of a CDW order when $n=1 / 2(\lambda=2)$. Another consideration is that, at the RPA level, the geometrical factor $\gamma(q) \propto \cos (\pi / \lambda)$ favors large $\lambda$. For $V_{\perp}>4$, the $\lambda=3$ curve eventually reaches the classical expectation. In addition to this feature, hole pairs have effective interactions at longer range ${ }^{11}$ which show up in the low $K$ that can be achieved. This should favor crystallization at small doping and we indeed see that the $\lambda=5$ and 4 curves are comparable.

Conclusion - The emergence of charge ordering in a 2D array of two-leg ladders with a zig-zag coupling mimicking the crystallographic structure of the ladder planes of SCCO has been studied using a MF approximation to treat the screened Coulomb interaction. Our numerical approach provides quantitative predictions for the order parameter of charge modulations with period $\lambda=3,4,5$. A good agreement with $\mathrm{LL}$ theory is found in the perturbative regime before reaching the classical limit. The analogy between the results for the two models suggests that such structures can be interpreted physically as pair density waves, a localization of Cooper pairs with no superconducting coherence, $\frac{18}{2}$ spatially organized as in a Wigner crystal of pairs. $\frac{6}{-}$ We note that only the $\lambda=3$ and 5 charge order have been found experimentally while our minimal model still supports a sizable order for the $\lambda=4$ modulation. An Hartree-Fock approach to a more sophisticated mode ${ }^{19}$ suggested a relative reduction of the $\lambda=4$ order but there is no clear suppression. Another proposal was to take into account a magnetic ring exchange ${ }^{7}$ that lowers the $\lambda=4$ charge order. However, according to the behavior of the pairing energy with doping and ring exchange in the t-J model,, 20 the $\lambda=5$ modulation should also be suppressed. A possible explanation could be that the experimental set-up was not able to probe the peculiar type of ordering of Fig. 1b which involves two inequivalent ladders. Lastly, the $\lambda=4$ pattern could be more sensitive to impurities, magnetic exchange, single particle hopping or pair tunneling between ladders leading to a competition with superconducting phases
${ }^{1}$ E. Dagotto and T. M. Rice, Science 271, 618 (1996).

2 M. Uehara et al., Journal of the Physical Society of Japan $\mathbf{6 5}$, 2764 (1996); H. E. K.M. Kojima, N. Motoyama and S. Uchida, J. Electron Spectrosc. Relat. Phenom. 117-118, 237 (2001).

${ }^{3}$ T. Fukuda, J. Mizuki, and M. Matsuda, Phys. Rev. B 66, 012104 (2002).

${ }^{4}$ S. van Smaalen, Phys. Rev. B 67, 026101 (2003).

5 P. Abbamonte et al., Nature 431, 1078 (2004).

${ }^{6}$ A. Rusydi et al., Phys. Rev. Lett. 97, 016403 (2006); A. Rusydi et al., Phys. Rev. Lett. 100, 036403 (2008).

7 A. Rusydi et al., Phys. Rev. B 75, 104510 (2007).

${ }^{8}$ S. R. White, I. Affleck, and D. J. Scalapino, Phys. Rev. B 65 , 165122 (2002).

${ }^{9}$ G. Roux, E. Orignac, S. R. White, and D. Poilblanc, Phys. Rev. B 76, 195105 (2007).

${ }^{10}$ S. R. White, Phys. Rev. Lett. 69, 2863 (1992); U. Schollwöck, Rev. Mod. Phys. 77, 259 (2005).

11 T. Siller, M. Troyer, T. M. Rice, and S. R. White, Phys. Rev. B 63, 195106 (2001).
${ }^{12}$ G. Roux, S. R. White, S. Capponi, and D. Poilblanc, Phys. Rev. Lett. 97, 087207 (2006).

13 T. Giamarchi, Quantum Physics in one Dimension International series of monographs on physics Vol. 121 (Oxford University Press, Oxford, UK, 2004).

${ }^{14}$ C. A. Hayward, D. Poilblanc, R. M. Noack, D. J. Scalapino, and W. Hanke, Phys. Rev. Lett. 75, 926 (1995).

15 S. Capponi and D. Poilblanc, Phys. Rev. B 66, 180503(R) (2002).

${ }^{16}$ K. Wohlfeld, A. M. Oleś, and G. A. Sawatzky, Phys. Status Solidi B 247, 668 (2010); private communication (2010).

17 H. J. Schulz and C. Bourbonnais, Phys. Rev. B 27, 5856 (1983); S. T. Carr and A. M. Tsvelik, Phys. Rev. B 65, 195121 (2002).

${ }^{18}$ H.-D. Chen, O. Vafek, A. Yazdani, and S.-C. Zhang, Phys. Rev. Lett. 93, 187002 (2004).

${ }^{19}$ K. Wohlfeld, A. M. Oleś, and G. A. Sawatzky, Phys. Rev. B 75, 180501 (2007).

${ }^{20}$ G. Roux, S. R. White, S. Capponi, A. Läuchli, and D. Poilblanc, Phys. Rev. B 72, 014523 (2005).

${ }^{21}$ In addition to numerical checks, we can argue that in the pertur- 
bative regime the $\lambda=4$ staggered pattern is stabilized w.r.t. the stripes configuration as the interaction energy difference (per site) is $e_{\text {stagg }}-e_{\text {int }} \propto-\rho_{q}^{2}(\sqrt{2}-1)$. In the classical limit where there is one particle each 4 site in (i), we see that the interaction energy is zero in the staggered pattern while it is positive for the stripes.

${ }^{22}$ The density patterns do not require a lot of states to converge, particularly when $V_{\perp} \neq 0$. For $V_{\perp}=0$, we checked using up to 1600 states.

${ }^{23}$ In the non-interacting limit where $K=1$, a BCS-like opening $\Delta \propto e^{- \text {const. } / V_{\perp}}$ is expected at small $V_{\perp}$. 